\title{
Cartas
}

\section{Estado de la normativa chilena que regula el uso de las radiaciones ionizantes en procedimientos de intervencionismo cardiológico}

\author{
Carlos Ubeda ${ }^{1 a}$, Patricia Miranda ${ }^{2}$, Daniel Aguirre $^{3}$, Luis Cárdenas $^{2,4}$, Nemorino Riquelme $^{3 b}$ y Anamaría Álvarez ${ }^{4 c}$. \\ 1. Departamento de Tecnología Médica, Laboratorio de Dosimetría personal, Facultad de Ciencias de la Salud, Universidad de Tarapacá. \\ 2. Servicio de Hemodinamia, Servicio Cardiovascular, Hospital Luis Calvo Mackenna. \\ 3. Servicio de Hemodinamia, Servicio Cardiovascular, Hospital Roberto del Río. \\ 4. Servicio de Hemodinamia, Servicio Cardiovascular, Clínica Santa María. \\ a. Tecnólogo Médico de Radiología con mención en Imagenología Física Médica. Licenciado en Ciencias Biológicas. Magíster en Biofísica Médica. \\ Doctor en Ciencias Radiológicas. \\ b. Tecnólogo Médica con mención en Imagenología y Radioterapia. Magíster en Ciencias Radiológicas. \\ c. Tecnólogo Médica con mención en Imagenología y Física Médica. Licenciada en Imagenología.
}

\section{Estimado Sr. Editor}

Resulta algo paradójico y a su vez, muy preocupante, que los avances tecnológicos de los equipos de rayos $\mathrm{X}$ utilizados para realizar los procedimientos de intervencionismo cardiológico denominados angiógrafos, hayan tenido un desarrollo acelerado y permanente en los últimos 25 años, mientras que la normativa nacional que regula el uso de estos equipos generadores de radiaciones ionizantes y sus aplicaciones en intervencionismo cardiológico, tengan una data del siglo pasado ${ }^{1,2}$.

Además, las aplicaciones en el diagnóstico y el tratamiento de las enfermedades han generado que el uso de las radiaciones ionizantes en medicina sea la principal fuente de exposición a la radiación creada por el ser hu- mano en la actualidad ${ }^{3}$. Los procedimientos de intervencionismo cardiológicos son potencialmente responsables de los más elevados niveles de radiación para pacientes y personal médico ${ }^{4}$. Adicional preocupación se debería prestar cuando estos pacientes son niños debido a su elevada radiosensibilidad y mayor esperanza de vida para manifestar alguna patología radioinducida ${ }^{3}$. Dada esta situación, el Organismo Internacional de Energía Atómica (OIEA) en conjunto con otras organizaciones vienen promoviendo buenas prácticas para la utilización de estas energías como los rayos $\mathrm{X}$, a través de su programa de normas de seguridad desde el año 1958. A partir del año 1996 incorpora requisitos internacionales para la exposición médica ${ }^{5}$, siendo su más vigente versión la publicada en el año $2016^{6}$. Por lo tanto, se hubiera esperado que

Correspondencia:

TM. Carlos Ubeda.

carlos.ubeda.uta@gmail.com. 
los países como Chile, miembro activo de las Naciones Unidas y por lo tanto del OIEA, debería haber incorporar en un tiempo prudente estas nuevas recomendaciones a su normativa nacional. En este sentido, la Directiva 2013/59/Euratom ${ }^{7}$, es un excelente ejemplo de lo que debemos hacer como país.

Desde el año $2007^{8-18}$ nuestro grupo de trabajo ha venido sistemáticamente aportando evidencia científica en revistas de corriente principal sobre la necesidad de actualizar la normativa nacional, que permita incorporar conceptos sobre: Programas de Garantía de Calidad y Protección Radiológica, Controles de Calidad, Comisionamiento de Angiógrafos, Especialistas en Física Médica, Formación en Protección Radiológica de Segundo Nivel para Médicos Cardiólogos Intervencionistas, Niveles de Referencia para Diagnóstico, Dosis Absorbida, Dosis Efectiva y otras magnitudes con sus respectivas unidades, etc.

Por todo lo anterior, resulta clave y urgente, conocer en que está la consulta pública del año 2017, sobre el nuevo Reglamento de Protección Radiológica propuesto por la división de políticas públicas saludables y promoción de la Subsecretaría de Salud Pública de Chile ${ }^{19}$ ya que fuimos muchos los que vimos en dicha iniciativa legal una luz de esperanza para mejorar la seguridad de nuestros pacientes y personal médico, durante estos procedimientos intervencionistas y otros que usen radiaciones ionizantes.

\section{Agradecimientos}

El investigador Carlos Ubeda agradece el apoyo de la Dirección de Investigación de la Universidad de Tarapacá, a través del proyecto de investigación UTA Mayor $\mathrm{N}^{\mathrm{o}}$ 7713-18.

\section{Bibliografía}

1. Reglamento de protección radiológica de instalaciones radiactivas, Decreto Supremo Nº3 de la República de Chile, 3 de Enero de 1985.

2. Reglamento sobre autorizaciones para instalaciones radioactivas o equipos generadores de radiaciones ionizantes, personal que se desempeña en ellas, u opere tales equipos y otras actividades afines, Decreto Supremo №133 de la República de Chile, 22 de mayo de 1984.

3. UNSCEAR. Sources and Effects of Ionizing Radiation. Volume I: Sources: Report to the General Assembly, Scientific Annexes A and B. UNSCEAR 2008 Report. United Nations Scientific Committee on the Effects of Atomic Radiation. United Nations sales publication E.10.XI.3. United Nations, New York, 2010.

4. ICRP. International Commission on Radiological Protection. Radiological protection in cardiology. ICRP Publication 120. Ann. ICRP 2013; 42.
5. IAEA. International Atomic Energy Agency, International Basic Safety Standards for Protection against Ionizing Radiation and for the Safety of Radiation Sources. Safety Series N ${ }^{\circ} .115$, IAEA, Vienna (1996).

6. DISPONIBLE EN: https://servei.org/wp-content/uploads/ P1578_S_web.pdf (accedido el 09 de febrero de 2020).

7. DISPONIBLE EN: http://www.medicosypacientes.com/sites/ default/files/euraton_2013.pdf (accedido el 18 de febrero de 2020).

8. UBEDA C, LEYTON F, GALAZ S, OYARZÚN C, INZULZA A. Garantía de calidad y protección radiológica en las exposiciones médicas en europa: un ejemplo a seguir. Rev Chil Radiol 2007;13(4): 208-212.

9. VANO E, UBEDA C, LEYTON F, MIRANDA P. Radiation dose and image quality for paediatric interventional cardiology. Phys Med Biol 2008;53(15):4049-4062. 
10. UBEDA C, VANO E, MIRANDA P, LEYTON F, VALENZUELA E, OYARZUN C. Radiation dose and image quality for adult interventional cardiology in Chile: a national survey. Radiat Prot Dosim 2011;147(1-2):90-93.

11. UBEDA C, MIRANDA P, VAÑO E, NOCETTI D. Protección radiológica en cardiología intervencionista Pediátrica. Avances y desafíos para Chile. Rev Chil Car 2013;32(3):223-229.

12. MIRANDA P, UBEDA C, VAÑO E, NOCETTI D. Recomendaciones para mejorar la seguridad radiológica durante los procedimientos de intervencionismo cardiológico. Rev Chil Car 2014;33(1):44-50.

13. UBEDA C, VAÑO E, RUIZ CRUCES R, SOFFIA P, FABRI D Niveles de referencia para diagnóstico: Una herramienta efectiva para la protección radiológica de pacientes. Rev Chil Radiol 2019; 25(1): 19-25.

14. UBEDA C, NOCETTI D, ALARCÓN R, INZULZA A, CALCAGNO S, CASTRO $M$ et al. Magnitudes y unidades para dosimetría a pacientes en radiodiagnóstico e intervencionismo. Rev Chil Radiol 2015;21(3):94-99.

15. CARLOS UBEDA DE LA C, DIEGO NOCETTI G, ALONSO
INZULZA C, CARLOS OYARZÚN C, RENATO ALARCÓN E. Magnitudes y unidades para dosimetría del personal ocupacionalmente expuesto en radiodiagnóstico e intervencionismo. Rev Chil Radiol 2018; 24(1):5-11.

16. UBEDA C, MIRANDA P, VANO E. Local patient dose diagnostic reference levels in pediatric interventional cardiology in Chile using age bands and patient weight values. Med Phys 2015;42(2):615-622

17. UBEDA C, VANO E, MIRANDA P, FIGUEROA X. Organ and effective doses detriment to paediatric patients undergoing multiple interventional cardiology procedures. Phys Med 2019; 60:182-187.

18. UBEDA C, VANO E, RIQUELME N, AGUIRRE D, VASQUEZ H, CHAVEZ C, DALMAZZO D. Patient radiation doses in paediatric interventional cardiology and optimization actions. Radiation Physics and Chemistry 2020; 168:108539

19. DISPONIBLE EN: https://www.minsal.cl/wp-content/ uploads/2017/01/Consulta-p\%C3\%BAblica-Reglamento-de-Protecci\%C3\%B3n-Radiol\%C3\%B3gica.pdf (accedido el 03 de marzo de 2020). 